\title{
The Spectrum of the Diseased Aortic Wall. Dissection and other Acute Aortic Syndromes...
}

Kotsis TE

From a holistic point of view, the most catastrophic diseases involving the aortic wall are the acute aortic syndromes (AAS) which include the penetrating aortic ulcer (PAU), the intramural hematoma (IMH) and aortic dissection (AD); the thoracic aorta is most frequently affected. The PAU is a localized form of aortic wall inner erosion where a crater is developed, and the IMH is a contained hematoma within the media, inside the tunica adventitia, without obvious communication with the aortic lumen; intramural hematomas, are developed from bleeding of the penetrating feeding vessels running through the aortic wall; these feeders are abundant in the thoracic aorta. The principal devastating arterial catastrophe, the dissection of the aortic wall with a blood entry point through an intimal tearing, results in the creation of a false lumen which travels through the tunica media; it is either developed following PAU or IMH or it emerges suddenly as an extended aortic disaster; the three aforementioned types of AAS have overlapping pathophysiology and may lead to false aneurysms and/or rupture with exsanguination. Different classifications of the AAS, and in particular of aortic dissection, have been elaborated based on the aortic injury extension, the risk of ischemia for vital organs and the chronicity; therapy is customized, on a case by case basis taking into account the complications and the anatomical section/s of the diseased aorta and its branches that are involved. The most vulnerable anatomical sites for the development of life threatening $\mathrm{AD}$ are the ascending aorta (70\%), the aortic arch $(7 \%)$ and the descending aorta (20\%). Immediate intervention is mandated when the entry tear of the aortic wall dissection is located proximal to the origin of the left subclavian artery; in this case, emergency surgery, mostly engaging extracorporeal circulation, is necessary, because of the high mortality observed in patients without repair; the death rate is $1 \%$ for every hour for the first 48 hours. When the dissection initiates distal to the origin of the left

\footnotetext{
Kotsis TE

Vascular Surgeon, Associate Professor of Medical School, National and Kapodistrian, University of Athens-Greece, Head of the Vascular Unit, $2^{\text {nd }}$ Department of Surgery, Aretaieion University Hospital
}

Corresponding author: Kotsis E Thomas

Vascular Surgeon, Associate Professor of Medical School, National and Kapodistrian, University of Athens-Greece, Head of the Vascular Unit, $2^{\text {nd }}$ Department of Surgery, Aretaieion University Hospital e-mail: kotsisth@otenet.gr subclavian artery, symptomatic patients or with intractable pain or high arterial pressure need to be managed either by catheter based techniques or open surgery.

On the other hand, isolated dissection of the abdominal aorta is rare; the reported rate of primary abdominal aortic dissection (AAD) is less than $2 \%$, compared with that of the thoracic aorta. An interesting review concerning this entity is reported in the next pages of this journal [1]; in an extended search of the world literature, the authors found that the AAD is observed more often in men than women (3:1), is acute in $72 \%$, spontaneous in $87.5 \%$, traumatic in $6.25 \%$ and iatrogenic in a good $6.25 \%$ of the patients; in $57.2 \%$ of the cases the entry point was located distal to the origin of renal arteries.

Although concurrent aortic aneurysms could be associated with spontaneous $\mathrm{AAD}$ at a rate of $27 \%$, the anatomical and clinical features of isolated AADs are different from those of the common abdominal aortic aneurysms.

Apart from evidence of known connective tissue diseases, characteristic atherosclerotic changes of the aortic wall also consist points of suspicion for AAD in the analog clinical setting; specific epidemiological features delineate the profile of a patient with the abdominal aortic syndrome. The younger patients with manifestations of peripheral occlusive disease, including coronary artery disease, are candidates for spontaneous or iatrogenic disrupture of atheromatic plaques and subsequent isolated, localized or more extended aortic syndromes. The older patients usually have hypertension, aneurysms, and aged aortas with a deteriorated aortic wall, and are prone to more severe aortic syndromes with increased extension and volume of the false lumen, and dissecting pseudoaneurysm formation.

Due to the current intense imaging of the human body and the frequent catheter based interventions, limited or extended aortic dissections are identified and reported now at a higher frequency than in the past. Modern imaging devices offer better resolution with minimization of voxels, which helps in identifying even miniscule tears of the aortic wall. Thoracic aortas with PAUs, IMHs and dissections are studied more thoroughly; intervention is mandated in all symptomatic patients, and earlier in those with connective tissue disorders; maximum PAU diameter exceeding $20 \mathrm{~mm}$ and depth of more than $10 \mathrm{~mm}$ both correlate significantly with advanced disease; also, when a false lumen becomes aneurysmal and exceeds $3 \mathrm{~cm}$, intervention needs to be considered. In the case of AADs, most authors recommend 
an aggressive strategy with similar indications. Symptomatic and patients with vital organ or limb ischemia and those with a concurrent aneurysm are favored for early intervention, although no specific threshold has been suggested. In case of pseudoaneurysm formation, especially following infrarenal dissection, a lower threshold for repair can be justified.

The current armamentarium for managing thoracic and abdominal aortic syndromes, and in particular the dreaded aortic dissection, is vast, with many customized options employing either open surgery, endovascular procedures or combinations of both. In this issue, Dodos et al review thoroughly the reported strategy for managing specifically patients with $\mathrm{AAD}$ [1]. Dissected aortas represent the utmost scenario of arterial wall disaster; medicine along with science and technology have, on the one hand to offer a biological solution for the remodelling of the diseased aortic wall, and on the other hand to support the spinal cord supply in extended dissections where the risk of paraplegia, although recently minimized, it cannot be eliminated despite the application of all modern adjuncts.

\section{References}

1. Dodos I, Theodoridis P, Staramos D, et al. Endovascular Repair of Isolated Abdominal Aortic Dissection. Literature Review. Hellenic J Surg 2018;90:85-89. 